\title{
Multidrug-resistant cytomegalovirus infection in a pediatric stem cell transplantation patient
} \author{
Sarah Gillemot ${ }^{\mathrm{d}}$, Pierre Fiten ${ }^{\mathrm{d}}$, Ghislain Opdenakker ${ }^{\mathrm{d}}$, Robert Snoeck ${ }^{\mathrm{d}}$, \\ Geneviève Laureys ${ }^{\text {a }}$ \\ ${ }^{a}$ Department of Pediatric Hemato-Oncology and Stem Cell Transplantation, Ghent University Hospital, Ghent, Belgium \\ b Department of Clinical Chemistry, Microbiology and Immunology, Ghent University Hospital, Ghent, Belgium \\ ' School of Life Sciences, Hasselt University, Hasselt, Belgium \\ ${ }^{\mathrm{d}}$ Rega Institute for Medical Research, Department of Microbiology and Immunology, KU Leuven, Leuven, Belgium
}

Tiene Bauters ${ }^{a,}{ }^{*}$, Victoria Bordon ${ }^{\text {a }}$, Lisa Florin ${ }^{\text {b }}$, Elizaveta Padalko ${ }^{\text {b, c }}$, Graciela Andrei ${ }^{\mathrm{d}}$

\section{A R T I C L E I N F O}

\section{Article history:}

Received 8 March 2016

Received in revised form 12 May 2016

Accepted 26 May 2016

Available online 11 June 2016

\section{Introduction}

Cytomegalovirus (CMV) is a member of the Herpesviridae family and is characterized by a lifelong latency in the host (Russell et al., 2011). Clinical presentations of CMV infection are mainly minimal to absent in immunocompetent hosts but can lead to severe symptoms up to life-threatening conditions in immunosuppressed patients.

Prophylactic or preemptive therapy against CMV infection has successfully reduced the incidence of CMV disease in transplant recipients (Bordon et al., 2008). However, it still remains one of the major infectious complications and causes of morbidity and mortality after hematopoietic stem cell transplantation (HSCT) (Russell et al., 2011; Ljungman et al., 2011). Risk factors for CMV infection in HSCT patients include CMV seropositivity of donor and recipient, allogeneic transplantation (especially with $\mathrm{T}$ cell-depleted unrelated or HLA-mismatched donors) and cell source. Cord blood recipients have both longer and higher cumulative incidences of CMV infection compared to peripheral blood or allogeneic bone marrow recipients from related or unrelated donor origin (Keever-Taylor et al., 2001; Yoon et al., 2009; Yi and Kim, 2012). In HSCT

\footnotetext{
* Corresponding author. Dept. of Pediatric Hemato-Oncology and Stem Cell Transplantation, Ghent University Hospital, De Pintelaan 185, B-9000, Ghent, Belgium.

E-mail address: tiene.bauters@uzgent.be (T. Bauters).
}

patients, cellular immunity is mainly significantly impaired during the first 100 days post-transplant. CMV infection in HSCT patients manifests as enterocolitis, pneumonia, hepatitis or as retinitis and bone marrow suppression (Russell et al., 2011). Although less frequently, late CMV reactivation ( $>100$ days posttransplant) can occur (Cervera et al., 2007; Sousa et al., 2014).

The number of available drugs for treatment of CMV infection is limited. Ganciclovir, the mainstay treatment of CMV infection, is a nucleoside analog that inhibits the viral DNA polymerase. It is first phosphorylated to its monophosphate form by the CMV-encoded UL97 protein kinase and further converted by cellular kinases to the triphosphate form, which is the active metabolite. Ganciclovirtriphosphate competitively inhibits the binding of deoxyguanosine triphosphate to the viral DNA polymerase finally resulting in inhibition of viral DNA synthesis (Biron, 2006). Ganciclovir remains the first-line agent for CMV prophylaxis and preemptive therapy reducing the risk of $C M V$ infection and disease compared to placebo (Ljungman et al., 2011). A major adverse effect related to its use is hematologic toxicity.

Other antiviral agents such as foscarnet and cidofovir, also inhibit the CMV DNA polymerase but have the ability to inhibit UL97 protein kinase mutants of CMV because cidofovir is independent of this kinase for activation and foscarnet does not require activation to inhibit the viral DNA polymerase (Biron, 2006). Their use is limited due to toxicity, more specifically to mineral and electrolyte abnormalities as a result of renal impairment (foscarnet) and renal toxicity associated with cidofovir. This underscores the importance of adequate hydration and frequent monitoring of serum creatinine levels in patients receiving foscarnet or cidofovir (Biron, 2006).

Resistance of CMV to ganciclovir is mostly caused by mutations within the UL97 gene and less frequently by alterations within the viral UL54 DNA polymerase gene (Gilbert and Boivin, 2005; Lurain and Chou, 2010). Resistance to cidofovir and foscarnet is linked to mutations in the viral DNA polymerase gene. In general, mutations conferring resistance to both cidofovir and ganciclovir are located 
in the exonuclease domain and region $\mathrm{V}$ of the viral DNA polymerase, whereas those conferring foscarnet resistance map between regions II and III of the viral enzyme. Some mutations in and near region III confer dual foscarnet and ganciclovir resistance, and a few mutations (such as A834P and deletion 981-2) confer resistance to the three anti-CMV drugs. Emergence of cidofovir resistance appears to be an extremely rare event and it arises in the clinic following sustained ganciclovir administration (Gilbert and Boivin, 2005; Lurain and Chou, 2010).

A few reports have highlighted the importance of CMV resistance in pediatric HSCT populations. CMV-infected infants after HSCT were found to develop ganciclovir resistance associated with the UL97 protein kinase (Ohta et al., 2001; Gohring et al., 2009; Kim et al., 2012; Erice et al., 1989). However, in some cases, multiple UL97 and UL54 (DNA polymerase) mutations that conferred resistance to ganciclovir and foscarnet (Choi et al., 2014) or to all three currently available anti-CMV drugs (i.e. ganciclovir, cidofovir and foscarnet) have been described (Blackman et al., 2004; Drouot et al., 2014; Springer et al., 2005).

We report a case of fatal CMV infection in a young patient with primary immunodeficiency and consecutive HSCT who developed first ganciclovir resistance (due to a mutation in the UL97 protein kinase) and later on multidrug-resistant CMV disease associated with a single mutation in the DNA polymerase.

\section{Materials and methods}

\subsection{Case}

A 4-month old girl (weight $5.8 \mathrm{~kg}$ ) was referred for fever, failure to thrive and bloody diarrhea. She was the first child of consanguineous parents, so severe immunodeficiency was presumed. CMV was detected in the blood with a viral load of $3.7710 \mathrm{E} 6$ copies/ $\mathrm{ml}$, treatment with ganciclovir (5 $\mathrm{mg} / \mathrm{kg}$, twice daily) was started. MHC class II deficiency was diagnosed and the search for a stem cell donor was initiated. Four weeks later the dose frequency was reduced to a once daily administration. However, 1 week later, the CMV viral load started to increase and therapy with ganciclovir was switched from once daily again to twice daily administration (double dose, based on the most recent body weight). As the CMV viral load still increased, ganciclovir administration was stopped and therapy was switched to foscarnet. No HLA identical donor nor cord blood was found so haploidentical HSCT was planned. For more than one month, the patient received foscarnet $(2 \times 350 \mathrm{mg})$ and simultaneously CMV-specific immunoglobulins. Antiviral resistance testing in a blood sample (RV-511) was performed and revealed the presence of a mixed population of M460V-mutant and wild-type virus in the UL97 protein kinase. This UL97 mutation is known to confer resistance to ganciclovir with susceptibility to foscarnet and cidofovir (Lurain and Chou, 2010). Results on genotyping testing of samples recovered from blood, cerebrospinal fluid (CSF) or nasopharyngeal aspirate (NPA) are presented in Table 1.

Despite antiviral treatment the patient deteriorated and developed CMV encephalitis confirmed by positivity of CMV PCR on CSF taken on day -14 (RV-517), which showed a similar resistance profile as the blood samples (RV-511 and RV-516) and the NPA (RV910) taken during the same time period. Retrospective analysis of NPA samples (RV-905 to RV-910) recovered before indicated that ganciclovir-resistance emerged at the moment that the patient developed encephalitis.

An allogeneic transplantation was performed on day zero (i.e. the day of HSCT) with peripheral blood stem cells from a haploidentical donor, after selection of CD34 cells from the graft.

Both the donor and receptor were CMV IgG seropositive. The conditioning regimen consisted of intravenous busulfan $(16 \times 1 \mathrm{mg} /$ $\mathrm{kg})$ and fludarabine $\left(4 \times 35 \mathrm{mg} / \mathrm{m}^{2}\right)$. Three days after HSCT, the patient developed fever for which antibiotics were started. Because of progression of neurologic encephalitic disturbances suggestive of central CMV infection, prophylactic anti-epileptics were started (phenobarbital) and cidofovir was added empirically to the foscarnet antiviral treatment. Antiviral resistance testing of a blood sample on day +1 (RV-520) showed, in addition to a mixed population of M460V-mutant and wild-type virus at the UL97 protein kinase, also the presence of a mixed population of 981-982 deletion mutant and wild-type virus in the DNA polymerase. This is known to confer resistance to ganciclovir, foscarnet and cidofovir (Lurain and Chou, 2010). The same viral populations were detected at the same time in the NPA (RV-911) on day +8 . (RV-521).

On day +19 , the UL97 M460V-mutant population was not identified anymore in the blood (RV-523) but a mixed population of DNA polymerase mutant virus bearing the 981-982 deletion was present. In only 3 days, the mutant virus totally replaced the wildtype virus (RV-525) and presented a pure population of DNA polymerase mutant virus with the 981-982 deletion. Thirteen days after HSCT, the patient was referred to the intensive care unit because of exacerbation of respiratory problems. Cidofovir was stopped because of severe cytopenia and increase of the CMV viral load. During the following days there was a further clinical deterioration of respiratory and liver function and uncontrollable sepsis. It was decided to stop antiviral treatment and to start palliative care. The presence of multidrug-resistant CMV infection [due to the presence of the 981-982 deletion in the viral DNA polymerase not only in the blood (RV-526, RV-528, and RV-527) but also in urine (RV-524 and RV-913) and NPA (RV-912)] together with encephalitis, were all cumulating in a respiratory arrest with cardiac arrest and death of the patient at day +36 . A schematic representation of the evolution of the CMV viral load and of the administration of anti-CMV therapy is presented in Fig. 1.

\subsection{CMV viral load determination}

Throughout the patient's treatment, CMV DNA monitoring was performed with quantitative CMV PCR. CMV PCR was performed following an in-house method based on real-time technology with continuous TaqMan-probe detection according to previously published protocol (Vanpoucke et al., 1999). The detection threshold was set at 300 copies $/ \mathrm{mL}$. Nucleic acid extraction was performed from whole blood following generic protocol on NucliSENS ${ }^{\circledR}$ easy$\operatorname{Mag}^{\mathbb{B}}$ (bioMerieux, Marcy-l'Etoile, France). Consecutive amplification and detection was performed using CFX96 Real-Time PCR Detection System (Bio-Rad Laboratories, Nazareth Eke, Belgium).

\subsection{Antiviral resistance for $C M V$}

Testing of CMV drug-resistance in the different isolates was performed by genotyping the UL97 protein kinase responsible for ganciclovir phosphorylation and the UL54 (viral DNA polymerase), as detailed in the RegaVir project (website: regavir.org). The DNA from the different clinical samples was first extracted with a QIAamp bloodkit (Qiagen) according to the manufacturer's instructions. The entire CMV UL54 and UL97 genes were amplified using FastStart high-fidelity DNA polymerase (Roche Applied Science). The polymerase chain reaction products were purified with a QIAquick Purification Kit (Qiagen), and gene amplicons were directly sequenced using the cycle sequencing BigDye Terminator kit, version 3.1, on an ABI 3730 sequencing system (Applied Biosystems) and a set of primers spanning the entire coding regions of the genes. The sequencing results were computer assembled and compared with the UL97 protein kinase and DNA polymerase sequences from the reference AD-169 CMV strain (Genbank accession 
Table 1

Overview of amino acids changes in UL97 and UL54 with resistance profiles.

\begin{tabular}{|c|c|c|c|c|c|c|c|c|}
\hline \multirow[t]{2}{*}{ Day } & \multirow[t]{2}{*}{ Samples (RegaVir code) } & \multirow[t]{2}{*}{ CMV DNA } & \multirow[t]{2}{*}{ Type of sample } & \multicolumn{2}{|c|}{ Amino acids changes related to resistance in: } & \multirow[t]{2}{*}{ Resistance to: } & \multicolumn{2}{|c|}{$\begin{array}{l}\text { Amino acid changes related to genetic } \\
\text { polymorphisms }\end{array}$} \\
\hline & & & & UL97 (protein kinase) & UL54 (DNA polymerase) & & UL97 (protein kinase) & UL54 (DNA polymerase) \\
\hline-82 & Not available & $3.77 \times 10 \mathrm{e} 6$ & Blood & Not available & Not available & Not available & Not available & Not available \\
\hline-79 & $R V-905$ & Positive & NPA & None & None & None & None & \\
\hline-72 & $R V-906$ & Positive & NPA & None & None & None & None & d \\
\hline-65 & $R V-907$ & Positive & NPA & None & None & None & None & d \\
\hline-43 & $R V-908$ & Positive & NPA & None & None & None & None & d \\
\hline-37 & $R V-909$ & DL & NPA & NA & NA & - & NA & NA \\
\hline-19 & $R V-910$ & Positive & NPA & $\mathrm{M} 460 \mathrm{~V}^{\mathrm{b}}$ & None & GCV & None & d \\
\hline-16 & RV-511 & $1.34 \times 10 \mathrm{e} 5$ & Blood & $\mathrm{M} 460 \mathrm{~V}^{\mathrm{b}}$ & none & GCV & None & d \\
\hline-14 & RV-517 & Positive & CSF & $\mathrm{M} 460 \mathrm{~V}^{\mathrm{b}}$ & none & GCV & None & d \\
\hline-9 & RV-516 & $1.44 \times 10 \mathrm{e} 5$ & Blood & $\mathrm{M} 460 \mathrm{~V}^{\mathrm{b}}$ & none & GCV & None & d \\
\hline+1 & RV-520 & $5.98 \times 10 \mathrm{e} 5$ & Blood & $\mathrm{M} 460 \mathrm{~V}^{\mathrm{b}}$ & $981-982$ del. $^{\mathrm{b}}$ & GCV, CDV, FOS & None & d \\
\hline+5 & $R V-911$ & Positive & NPA & $\mathrm{M} 460 \mathrm{~V}^{\mathrm{b}}$ & $981-982$ del. $^{\mathrm{b}}$ & $\mathrm{GCV}, \mathrm{CDV}, \mathrm{FOS}$ & None & d \\
\hline & RV-521 & $5.52 \times 10 \mathrm{e} 6$ & Blood & $\mathrm{M} 460 \mathrm{~V}^{\mathrm{b}}$ & $981-982$ del. $^{\mathrm{b}}$ & GCV, CDV, FOS & N68D, I244V & d \\
\hline+19 & RV-523 & $1.06 \times 10 \mathrm{e} 7$ & Blood & none & 981-982 del. & GCV, CDV, FOS & None & d \\
\hline+22 & RV-525 & $1.65 \times 10 \mathrm{e} 6$ & Blood & none & $981-982$ del. $^{c}$ & GCV, CDV, FOS & None & d \\
\hline+26 & RV-526 & $5.94 \times 10 \mathrm{e} 7$ & Blood & none & $981-982$ del. $^{c}$ & GCV, CDV, FOS & None & d \\
\hline+28 & RV-524 & Positive & Urine & none & $981-982$ del. $^{c}$ & GCV, CDV, FOS & None & d \\
\hline+29 & RV-528 & $5.09 \times 10 \mathrm{e} 7$ & Blood & none & $981-982$ del. $^{\text {c }}$ & GCV, CDV, FOS & None & d \\
\hline+30 & $R V-913$ & Positive & Urine & none & 981-982 del. & GCV, CDV,FOS & None & d \\
\hline+31 & $R V-912$ & ND & NPA & none & $981-982$ del. $^{c}$ & GCV, CDV, FOS & None & d \\
\hline+33 & RV-527 & $1.29 \times 10 \mathrm{e} 8$ & Blood & none & 981-982 del. ${ }^{c}$ & $\mathrm{GCV}, \mathrm{CDV}, \mathrm{FOS}$ & None & d \\
\hline
\end{tabular}

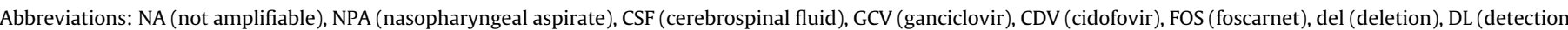
limit), ND (not detected).

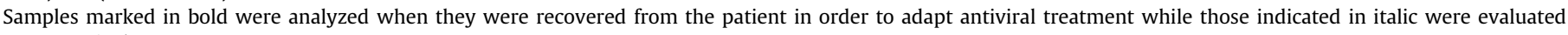
retrospectively.

a CMV DNA viral load (copies/ml) for blood or qualitative result for other specimen types.

b Mixed population of wild-type and mutant virus.

c Pure population of mutant virus.

d Amino acid changes in S655L, F669L, N685S, A885T and N898D.

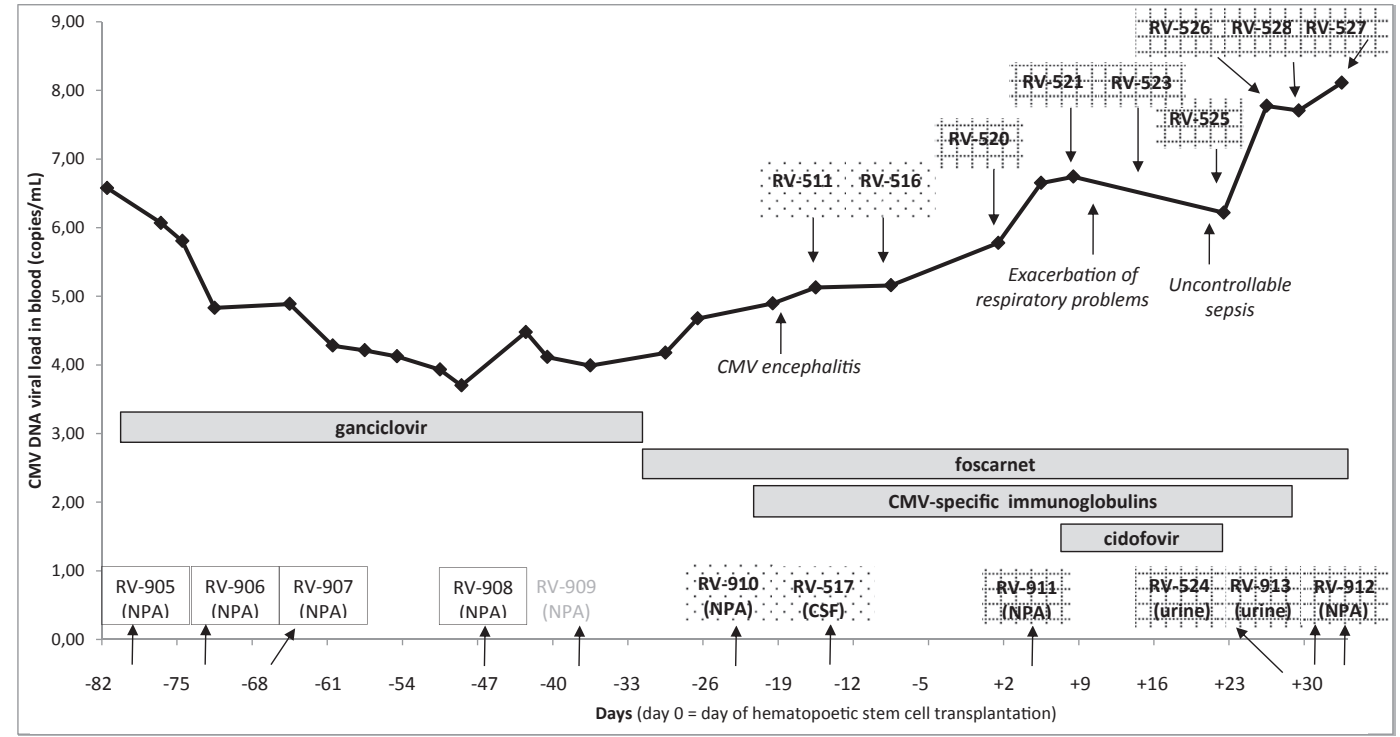

$\square$ Wild-type; $[$ Ganciclovir resistance; Ganciclovir, cidofovir, foscarnet resistance; The RV-909 (NPA) sample was not amplifiable Abbreviations: NPA (nasopharyngeal aspirate), CSF (cerebrospinal fluid)

Fig. 1. Overview of CMV-DNA PCR in relation to time, anti-CMV therapy and samples genotypically analyzed.

number of the reference sequence AD169 is X17403) using the software SeqScape, version 2.7 (Applied Biosystems). The presence of the mutations was confirmed by two independent sequencing reactions using both forward and reverse primers.

\section{Discussion}

Ganciclovir has been the mainstay antiviral drug for CMV treatment for many years. However, an increasing number of ganciclovir-resistant CMV infections has been reported in solid organ and especially pediatric bone marrow transplant recipients (Lurain and Chou, 2010; Strasfeld and Chou, 2010). Here we report a case of multidrug-resistant CMV infection in a severely immunocompromised pediatric patient. CMV encephalitis as a rare complication after HSCT was in our patient associated with recurrent CMV viremia following prolonged administration of 
ganciclovir and foscarnet and emergence of drug-resistance. The development of CMV central nervous system disease in HSCTpatients is associated with several risk factors, including, among others, drug-resistance and high mortality despite therapy with a combination of anti-CMV drugs (ganciclovir, foscarnet and cidofovir) (Reddy et al., 2010). Suboptimal ganciclovir dose may promote the emergence of drug-resistance since adequate drug levels are crucial to control viral replication. High drug levels are known to delay or prevent development of resistance while suboptimal drug doses encourage viral replication and emergence of drugresistance.

In contrast to the present case-report representing infection by the same CMV strain in different body compartments, a case of temporal and spatial compartmentalization of drug-resistant cytomegalovirus (CMV) in a child with CMV meningoencephalitis has been described by Frange et al. (Frange et al., 2013).

Rapid availability of CMV viral load measurements as well as sensitive detection of CMV in CSF are crucial for adequate antiviral chemotherapy (Boeckh, 2011). If resistance is suspected based on the persistently rising viral load, its accurate assessment by genotypic testing is mandatory (Boeckh et al., 2015). In the presented case report, close monitoring of genotypic resistance gave an opportunity to modulate the antiviral treatment in function of the identified mutant viruses. In our severely immunocompromised pediatric patient under antiviral drug pressure, a mixed population of wild-type and mutant virus bearing a point mutation in the UL97 protein kinase (M460V) was first detected in different body compartments (CSF, NPA and blood). This UL97 amino acid change is known to be associated with resistance to ganciclovir. After a short period, a new viral mutant harboring the DNA polymerase 981-982 deletion in the DNA polymerase was detected in addition to the UL97 mutant virus, both in the blood and NPA. Importantly, this DNA polymerase deletion has been linked to multiple drugresistance (ganciclovir, cidofovir and foscarnet). The DNA polymerase 981-982 deletion mutant appeared to emerge following foscarnet therapy in our patient (Fig. 1). Two weeks later, the presence of a pure mutant viral population with the deletion in UL54 was identified in the blood. Notably, the virus with the DNA polymerase deletion was later on detected not only in blood but also in urine and NPA, indicating a generalized multidrugresistance CMV infection. At this point, no approved therapeutic options for CMV disease were available and unfortunately, no access to compassionate use of novel anti-CMV drugs under clinical development was available. Although a few reports have described the use of leflunomide for therapy of multidrug-resistance CMV disease (Avery et al., 2004; Levi et al., 2006; Verkaik et al., 2013; Dunn et al., 2013), treatment with this drug having both immunosuppressive and anti-CMV properties was not envisaged in our patient.

Although new molecules (i.e. maribavir, letermovir, brincidofovir) are studied in ongoing clinical trials (www.clinicaltrials.gov; Sellar and Peggs, 2012), this case report indicates the urgent need for the development and market availability of new anti-CMV drugs. Although additional data are required, there might be role for artesunate in the treatment of mild CMV disease due to multidrug-resistant CMV strains (Germi et al., 2014).

Rapid modern molecular diagnostic approaches are essential for detection of CMV resistance as a guidance for treatment of transplant recipients at high risk for drug-resistance CMV disease.

\section{Author's contributions}

Concept/design: T. Bauters, V. Bordon, E. Padalko.

Data acquisition/analysis/interpretation and manuscript revision: T. Bauters, V. Bordon, L. Florin, E. Padalko, R. Snoeck, G. Andrei,
S. Gillemot, P. Fiten, G. Opdenakker, G. Laureys. Drafting article: T. Bauters, V. Bordon, E. Padalko, G. Andrei, R. Snoeck.

Approval of article: all authors.

\section{Acknowledgements}

Financial disclosure: no conflicts of interest or financial support exist.

\section{References}

Avery, R.K., Bolwell, B.J., Yen-Lieberman, B., Lurain, N., Waldman, W.J. Longworth, D.L., et al., 2004. Use of leflunomide in an allogeneic bone marrow transplant recipient with refractory cytomegalovirus infection. Bone Marrow Transpl. 34, 1071-1075.

Biron, K.K., 2006. Antiviral drugs for cytomegalovirus diseases. Antivir. Res. 71, $154-163$.

Blackman, S.C., Lurain, N.S., Witte, D.P., Filipovich, A.H., Groen, P., Schleiss, M.R. 2004. Emergence and compartmentalization of fatal multi-drug-resistant cytomegalovirus infection in a patient with autosomal-recessive severe combined immune deficiency. J. Pediatr. Hematol. Oncol. 26, 601-605.

Boeckh, M., 2011. Complications, diagnosis, management, and prevention of CMV infections: current and future. Hematol. Am. Soc. Hematol. Educ. Program 2011, 305-309.

Boeckh, M., Murphy, W.J., Peggs, K.S., 2015. Recent advances in cytomegalovirus: an update on pharmacologic and cellular therapies. Biol. Blood Marrow Transpl. 21, S19-S24.

Bordon, V., Bravo, S., Van Renterghem, L., de Moerloose, B., Benoit, Y., Laureys, G. et al., 2008. Surveillance of cytomegalovirus (CMV) DNAemia in pediatric allogeneic stem cell transplantation: incidence and outcome of CMV infection and disease. Transpl. Infect. Dis. 10, 19-23.

Cervera, C., Pineda, M., Linares, L., Marcos, M.A., Esteva, C., Anton, A., et al., 2007. Impact of valganciclovir prophylaxis on the development of severe latecytomegalovirus disease in high-risk solid organ transplant recipients Transpl. Proc. 39, 2228-2230.

Choi, S.H., Hwang, J.Y., Park, K.S., Kim, Y., Lee, S.H., Yoo, K.H., et al., 2014. The impact of drug-resistant cytomegalovirus in pediatric allogeneic hematopoietic cell transplant recipients: a prospective monitoring of UL97 and UL54 gene mutations. Transpl. Infect. Dis. 16, 919-929.

Drouot, E., Piret, J., Lebel, M.H., Boivin, G., 2014. Characterization of multiple cytomegalovirus drug resistance mutations detected in a hematopoietic stem cel transplant recipient by recombinant phenotyping. J. Clin. Microbiol. 52, 4043-4046.

Dunn, J.H., Weinberg, A., Chan, L.K., Mandava, N., Levi, M.E., Olson, J.L., 2013. Longterm suppression of multidrug-resistant cytomegalovirus retinitis with systemically administered leflunomide. JAMA Ophthalmol. 131, 958-960.

Erice, A., Chou, S., Biron, K.K., Stanat, S.C., Balfour Jr., H.H., Jordan, M.C., 1989. Progressive disease due to ganciclovir-resistant cytomegalovirus in immunocompromised patients. N. Engl. J. Med. 320, 289-293.

Frange, P., Boutolleau, D., Leruez-Ville, M., Touzot, F., et al., 2013 Dec. Temporal and spatial compartmentalization of drug-resistant cytomegalovirus (CMV) in a child with CMV meningoencephalitis: implications for sampling in molecular diagnosis. J. Clin. Microbiol. 51 (12), 4266-4269.

Germi, R., Mariette, C., Alain, S., Lupo, J., Thiebaut, A., et al., 2014 Jan. Success and failure of artesunate treatment in five transplant recipients with disease caused by drug-resistant cytomegalovirus. Antivir. Res. 101, 57-61.

Gilbert, C., Boivin, G., 2005. Human cytomegalovirus resistance to antiviral drugs. Antimicrob. Agents Chemother. 49, 873-883.

Gohring, K., Feuchtinger, T., Mikeler, E., Lang, P., Jahn, G., Handgretinger, R., et al., 2009. Dynamics of the emergence of a human cytomegalovirus UL97 mutant strain conferring ganciclovir resistance in a pediatric stem-cell transplant recipient. J. Mol. Diagn 11, 364-368.

Keever-Taylor, C.A.,Bredeson, C. Loberiza, F.R., Casper, J.T. Lawton, C. Rizzo, D. et al., 2001. Analysis of risk factors for the development of GVHD after T celldepleted allogeneic BMT: effect of HLA disparity, ABO incompatibility, and method of T-cell depletion. Biol. Blood Marrow Transpl. 7, 620-630.

Kim, Y.J., Boeckh, M., Cook, L., Stempel, H., Jerome, K.R., Boucek Jr., R., et al., 2012. Cytomegalovirus infection and ganciclovir resistance caused by UL97 mutations in pediatric transplant recipients. Transpl. Infect. Dis. 14, 611-617.

Levi, M.E. Mandava, N., Chan, L.K., Weinberg, A., Olson, J.L., 2006. Treatment of multidrug-resistant cytomegalovirus retinitis with systemically administered leflunomide. Transpl. Infect. Dis. 8, 38-43.

Ljungman, P., Hakki, M., Boeckh, M., 2011. Cytomegalovirus in hematopoietic stem cell transplant recipients. Hematol. Oncol. Clin. North Am. 25, 151-169.

Lurain, N.S., Chou, S., 2010. Antiviral drug resistance of human cytomegalovirus Clin. Microbiol. Rev. 23, 689-712.

Ohta, H., Matsuda, Y., Tokimasa, S., Sawada, A., Kim, J.Y., Sashihara, J., et al., 2001 Foscarnet therapy for ganciclovir-resistant cytomegalovirus retinitis after stem cell transplantation: effective monitoring of CMV infection by quantitative analysis of CMV mRNA. Bone Marrow Transpl. 27, 1141-1145. 
Reddy, S.M., Winston, D.J., Territo, M.C., Schiller, G.J., 2010. CMV central nervous system disease in stem-cell transplant recipients: an increasing complication of drug-resistant CMV infection and protracted immunodeficiency. Bone Marrow Transpl. 45, 979-984.

Russell, M.Y., Palmer, A., Michaels, M.G., 2011. Cytomegalovirus infection in pediatric immunocompromised hosts. Infect. Disord. Drug Targets 11, 437-448.

Sellar, R.S., Peggs, K.S., 2012. Management of multidrug-resistant viruses in the immunocompromised host. Br. J. Haematol. 156, 559-572.

Sousa, H., Boutolleau, D., Ribeiro, J. Teixeira, A.L., Pinho Vaz, C. Campilho, F. Branca, R., Campos Jr., A., Baldaque, I., Medeiros, R., 2014 Dec. Cytomegalovirus infection in patients who underwent allogeneic hematopoietic stem cel transplantation in Portugal: a five-year retrospective review. Biol. Blood Marrow Transpl. 20 (12), 1958-1967.

Springer, K.L., Chou, S., Li, S., Giller, R.H., Quinones, R., Shira, J.E., et al., 2005. How evolution of mutations conferring drug resistance affects viral dynamics and clinical outcomes of cytomegalovirus-infected hematopoietic cell transplant recipients. J. Clin. Microbiol. 43, 208-213.
Strasfeld, L., Chou, S., 2010. Antiviral drug resistance: mechanisms and clinical implications. Infect. Dis. Clin. North Am. 24, 809-833.

Vanpoucke, H., Van Vlem, B., Vanholder, R., Van Renterghem, L., 1999. Significance of qualitative polymerase chain reaction combined with quantitation of viral load in the diagnosis and follow-up of cytomegalovirus infection after solidorgan transplantation. Intervirology 42, 398-404.

Verkaik, N.J., Hoek, R.A., van, B.H., van Hal, P.T., Schipper, M.E., Pas, S.D., et al., 2013. Leflunomide as part of the treatment for multidrug-resistant cytomegalovirus disease after lung transplantation: case report and review of the literature. Transpl. Infect. Dis. 15, E243-E249.

Yi, E.S., Kim, Y.J., 2012. Cytomegalovirus infection according to cell source after hematopoietic cell transplantation in pediatric patients. Yonsei Med. J. 53, 393-400.

Yoon, H.S., Lee, J.H., Choi, E.S., Seo, J.J., Moon, H.N., Kim, M.N., et al., 2009. Cytomegalovirus infection in children who underwent hematopoietic stem cell transplantation at a single center: a retrospective study of the risk factors. Pediatr. Transpl. 13, 898-905. 